\title{
Comparison of the prognostic value of immunoinflammation- based biomarkers in patients with gastric cancer
}

\author{
Noriyuki Hirahara ${ }^{1}$, Takeshi Matsubara ${ }^{1}$, Yusuke Fujii ${ }^{1}$, Shunsuke Kaji ${ }^{1}$, \\ Yasunari Kawabata ${ }^{1}$, Ryoji Hyakudomi ${ }^{1}$, Tetsu Yamamoto ${ }^{1}$, Takahito Taniura ${ }^{1}$ \\ and Yoshitsugu Tajima ${ }^{1}$ \\ ${ }^{1}$ Department of Digestive and General Surgery, Shimane University Faculty of Medicine, Shimane 693-8501, Japan \\ Correspondence to: Noriyuki Hirahara, email: norinorihirahara@yahoo.co.jp \\ Keywords: gastric cancer; systemic immune-inflammation index; CRP; overall survival
}

Received: May 04, $2020 \quad$ Accepted: June 05, $2020 \quad$ Published: July 07, 2020

Copyright: Hirahara et al. This is an open-access article distributed under the terms of the Creative Commons Attribution License 3.0 (CC BY 3.0), which permits unrestricted use, distribution, and reproduction in any medium, provided the original author and source are credited.

\section{ABSTRACT}

Background: Systemic immune-inflammation index (SII)-comprising platelet, neutrophil, and Iymphocyte count-is an objective and reliable biomarker for predicting the prognosis in cancer patients because it comprehensively reflects the balance between host inflammatory and immune responses. In this study, we clarified the prognostic impact of immunoinflammation-based indices, i. e. SII, neutrophil/ lymphocyte ratio (NLR), and platelet/lymphocyte ratio (PLR), in gastric cancer patients.

Results: In multivariate analysis, the American Society of Anesthesiologists physical status (ASA-PS) (hazard ratio [HR]: $3.366, p<0.001$ ), tumor differentiation (HR: 1.705, $p=0.020$ ), pathological Tumor, Node, Metastasis (pTNM) stage (HR: 2.160, $p=0.008$ ), and carcinoembryonic antigen (CEA) (HR: 1.964, $p=0.003$ ) were independent prognostic factors for OS in all patients. Further, multivariate analysis revealed that age (HR: 2.088, $p=0.040$ ), ASA-PS (HR: 2.339, $p=0.043$ ), tumor differentiation (HR: 1.748, $p=0.044$ ), and pTNM stage (HR: 2.114, $p=0.024$ ) were independent prognostic factors for OS among patients without inflammation; SII was not a prognostic factor for OS. Meanwhile, body mass index (HR: 5.055, $p=0.011$ ), ASA-PS (HR: 3.403, $p=0.007$ ), and SII (HR: 4.208, $p=0.026$ ) were independent prognostic factors for OS among patients with inflammation.

Materials and Methods: We performed a retrospective review of 412 patients who underwent curative laparoscopic gastrectomy. The prognostic value of SII was compared between a low SII group (SII<661.9) and high SII group (SII $\geq 661.9$ ). We analyzed the predictive ability of immunoinflammation-based indices for overall survival (OS) based on a C-reactive protein (CRP) level of 0.5 .

Conclusions: Compared to NLR and PLR, SII is the most significant prognostic biomarker for OS, especially in gastric cancer patients with inflammation.

\section{INTRODUCTION}

Numerous studies have reported that cancer-related inflammation is an indispensable component of the tumor microenvironment. Cancer causes local or systemic inflammation, ultimately promoting cancer initiation and progression by escaping from the immune system [1-3]. Additionally, systemic immunoinflammation has been generally accepted to affect the cancer microenvironment in a way that favors proliferation, invasion, and migration of cancer cells, while reducing the response to anticancer agents.

Several systemic immunoinflammatory parameters have been evaluated as candidates for predicting longterm survival in various malignancies because systemic immunoinflammation is considered as a consequence, 
rather than the cause of cancer [4]. Recently, Hu et al. demonstrated that the systemic immune-inflammation index (SII) has a strong independent prognostic value in patients with hepatocellular carcinoma treated with surgery [5]. SII comprises 3 peripheral blood parameters, i. e., platelet, neutrophil, and lymphocyte count, which comprehensively reflect the balance of host immune and inflammatory status. In addition, SII is shown to be more objective and reliable for predicting survival in cancer patients than other hematological parameters, including neutrophil/lymphocyte ratio (NLR) and platelet/ lymphocyte ratio (PLR), in which both NLR and PLR are based on two inflammatory cells [6-8].

Currently, prognostic prediction in cancer patients mainly depends on the Tumor, Node, Metastasis (TNM) staging system, but the final TNM stage is defined by the histological evaluation of resected specimens after surgery [9]. Preoperative prognostic prediction remains difficult, and the definitive predictor of survival in cancer patients is a subject of ongoing debate. SII is an easily obtained, inexpensive, and non-invasive biomarker that could complement TNM stage in the preoperative prediction of survival in cancer patients. In this study, we clarified the prognostic impact of immunoinflammation-based indices, including SII as well as NLR and PLR, in gastric cancer patients who underwent curative laparoscopic gastrectomy.

\section{RESULTS}

\section{SII and clinicopathological features in overall patients}

The relationships between the SII values and clinicopathological characteristics in the 412 patients enrolled in this study are summarized in Table 1. Based on the SII cutoff value, 307 patients $(74.5 \%)$ and 105 patients (25.5\%) were classified as having a low SII and a high SII, respectively. SII was significantly associated with age $(p=0.024)$, American Society of Anesthesiologists physical status (ASA-PS) classification $(p<0.001)$, BMI $(p=0.044)$, white blood cell count (WBC) $(p<0.001)$, lymphocyte count $(p<0.001)$, neutrophil count $(p<0.001)$, platelet count $(p<0.001)$, tumor size $(p=0.002)$, depth of tumor $(p<0.001)$, lymph node metastasis $(p=0.044)$, pTNM stage $(p<0.001)$, C-reactive protein (CRP) level $(p<0.001)$, NLR $(p<0.001)$, and PLR $(p<0.001)$ (Table 1$)$.

\section{Cox regression analysis of OS in overall patients}

Univariate analyses revealed that worse OS was significantly associated with older age $(p=0.006)$, high BMI $(p=0.046)$, poor ASA-PS $(p<0.001)$, large tumor size $(p<0.001)$, poor differentiation $(p=0.004)$, advanced pTNM stage $(p<0.001)$, high carcinoembryonic antigen
(CEA) level $(p<0.001)$, high CRP level $(p<0.001)$, high SII $(p<0.001)$, high NLR $(p<0.001)$, high PLR $(p<0.001)$, postoperative complications (present) $(p=0.002)$, and postoperative adjuvant chemotherapy (yes) $(p=0.005)$. Multivariate analysis revealed that ASA-PS (HR: 3.366, 95.0\% CI: 1.917 - 5.911; $p<0.001$ ), tumor differentiation (HR: 1.705, 95.0\% CI: 1.087-2.674; $p=0.020)$, pTNM stage (HR: 2.160, 95.0\% CI: 1.218 $3.758 ; p=0.008$ ), and CEA (HR: $1.964,95.0 \% \mathrm{CI}: 1.251-$ $3.083 ; p=0.003)$ were the independent prognostic factors for OS (Table 2).

\section{SII and clinicopathological features in inflammation-stratified patients}

Based on a CRP level of $0.5 \mathrm{mg} / \mathrm{dl}, 350$ patients $(85.0 \%)$ were categorized into the non-inflammation group $(\mathrm{CRP}<0.5)$ and 62 patients $(15.0 \%)$ into the inflammation group $\left(\mathrm{CRP}^{3} 0.5\right)$. In patients without inflammation, 274 patients $(78.3 \%)$ were classified into the low SII group and the remaining 76 patients $(21.7 \%)$ were classified into the high SII group; the SII was significantly associated with ASA-PS $(p=0.044)$, WBC $(p<0.001)$, lymphocyte count $(p<0.001)$, neutrophil count $(p<0.001)$, platelet count $(p<0.001)$, tumor size $(p=0.015), \mathrm{CEA}(p=0.041), \mathrm{CRP}$ $(p=0.014)$, NLR $(p<0.001)$, and PLR $(p<0.001)$.

Among the 62 patients with inflammation, 33 patients $(53.2 \%)$ were classified into the low SII group and the remaining 29 patients $(46.8 \%)$ were classified into the high SII group. SII was significantly associated with lymphocyte count $(p=0.002)$, neutrophil count $(p<0.001)$, platelet count $(p<0.001)$, depth of tumor $(p=0.002)$, lymph node metastasis $(p=0.047), \mathrm{pTNM}$ stage $(p=0.001)$, CRP level $(p<0.001)$, NLR $(p<0.001)$, PLR $(p<0.001)$, and postoperative complications $(p=0.024)$ (Table 3$)$.

\section{Cox regression analysis of OS in inflammation- stratified patients}

In patients without inflammation, univariate analyses identified that a worse OS was significantly associated with older age $(p=0.011)$, poor ASA-PS $(p=0.002)$, large tumor size $(p<0.001)$, poor differentiation $(p=0.001)$, advanced pTNM stage $(p<0.001)$, high CEA level $(p=0.001)$, high SII $(p=0.002)$, high NLR $(p<0.001)$, high PLR $(p<0.001)$, and postoperative adjuvant chemotherapy (yes) $(p<0.001)$. Multivariate analysis revealed that age (HR: 2.088, 95.0\% CI: 1.033-4.221; $p=0.040$ ), ASA-PS (HR: 2.339, 95.0\% CI: 1.026-5.332; $p=0.043$ ), tumor differentiation (HR: 1.748, 95.0\% CI: 1.014-3.013; $p=0.044$ ), and pTNM stage (HR: 2.114, 95.0\% CI: $1.103-4.050 ; p=0.024)$ were the independent prognostic factors for OS (Table 4). The SII was not confirmed to be an independent prognostic factor for OS. 
Table 1: Relationships between SII and clinicopathological features in overall gastric cancer patients

\begin{tabular}{|c|c|c|c|c|}
\hline \multirow{3}{*}{ Characteristics } & \multicolumn{4}{|c|}{ SII } \\
\hline & \multirow{2}{*}{ Total patients } & $<661.9$ & $\geq 661.9$ & \multirow{2}{*}{$p$ value } \\
\hline & & $(n=307)$ & $(n=105)$ & \\
\hline Age (years) & & $70(36-91)$ & $74(43-90)$ & 0.024 \\
\hline Gender & & & & 0.648 \\
\hline Male & 287 & 212 & 75 & \\
\hline Female & 125 & 95 & 30 & \\
\hline ASA & & & & $<0.001$ \\
\hline 1 & 24 & 20 & 4 & \\
\hline 2 & 349 & 269 & 80 & \\
\hline 3 & 39 & 18 & 21 & \\
\hline BMI & & $22.5(14.7-40.4)$ & $21.8(14.0-32.5)$ & 0.044 \\
\hline WBC & & $5530(1830-9280)$ & $6490(3510-13700)$ & $<0.001$ \\
\hline Lymphocyte & & $1760(470-3780)$ & $1220(230-2500)$ & $<0.001$ \\
\hline Neutroohil & & $3169(1100-6190)$ & $4510(2650-8494)$ & $<0.001$ \\
\hline Platelet & & $205(58-460)$ & $251(119-726)$ & $<0.001$ \\
\hline Tumor size (mm) & & $40(3-180)$ & $50(5-170)$ & 0.002 \\
\hline Differentiation & & & & 0.305 \\
\hline Well & 81 & 65 & 16 & \\
\hline Moderate & 152 & 108 & 44 & \\
\hline Poor & 179 & 134 & 45 & \\
\hline Depth of tumor & & & & $<0.001$ \\
\hline T1a-1b & 218 & 180 & 38 & \\
\hline 2 & 54 & 42 & 12 & \\
\hline 3 & 59 & 37 & 22 & \\
\hline $4 a-4 b$ & 81 & 48 & 33 & \\
\hline Lymph node metastasis & & & & 0.044 \\
\hline N0 & 274 & 215 & 59 & \\
\hline N1 & 50 & 36 & 14 & \\
\hline $\mathrm{N} 2$ & 45 & 27 & 18 & \\
\hline N3 & 43 & 29 & 14 & \\
\hline Pathological stage & & & & $<0.001$ \\
\hline $1 a-1 b$ & 247 & 203 & 44 & \\
\hline $2 a-2 b$ & 73 & 49 & 24 & \\
\hline $3 a-3 c$ & 92 & 55 & 37 & \\
\hline CEA antigen (ng/ml) & & $3.2(0.7-106)$ & $3.5(0.8-163.3)$ & 0.144 \\
\hline CRP (mg/l) & & $0.07(0.01-6.31)$ & $0.15(0.01-11.10)$ & $<0.001$ \\
\hline NLR & & $1.826(0.648-6.893)$ & $\begin{array}{c}4.660 \\
(1.880-16.043)\end{array}$ & $<0.001$ \\
\hline PLR & & $116.9(43.8-276.9)$ & $212.0(99.4-992.7)$ & $<0.001$ \\
\hline Postoperative complications & & & & 0.087 \\
\hline absent & 290 & 223 & 67 & \\
\hline present & 122 & 84 & 38 & \\
\hline Adjuvant chemotherapy & & & & 0.133 \\
\hline Yes & 114 & 79 & 35 & \\
\hline No & 298 & 228 & 70 & \\
\hline
\end{tabular}


Table 2: Univariate and multivariate analyses for OS in overall gastric cancer patients

\begin{tabular}{lcccccccc}
\hline \multirow{2}{*}{ Variables } & Patients & Category or & \multicolumn{4}{c}{ Univariate } & \multicolumn{3}{c}{ Multivariate } \\
\cline { 5 - 9 } & $(\boldsymbol{n}=\mathbf{4 1 2})$ & characteristics & HR & $\mathbf{9 5 \% C I}$ & $\boldsymbol{p}$ value & HR & $\mathbf{9 5 \% C I}$ & $\boldsymbol{p}$ value \\
\hline Age & $112 / 300$ & $(<65 / \geq 65)$ & 2.242 & $1.268-3.962$ & 0.006 & 1.576 & $0.870-2.854$ & 0.134 \\
Gender & $125 / 287$ & $($ female/male) & 1.389 & $0.865-2.228$ & 0.174 & & & \\
BMI & $372 / 40$ & $(>18.5 /<18.5)$ & 1.785 & $1.009-3.156$ & 0.046 & 1.696 & $0.928-3.100$ & 0.086 \\
ASA & $373 / 39$ & $(<3 / \geq 3)$ & 4.378 & $2.613-7.336$ & $<0.001$ & 3.366 & $1.917-5.911$ & $<0.001$ \\
Tumor size & $244 / 168$ & $(<5 / \geq 5)$ & 2.275 & $1.496-3.460$ & $<0.001$ & 1.396 & $0.836-2.332$ & 0.202 \\
Differentiation & $232 / 180$ & well \& mod/poor & 1.85 & $1.220-2.807$ & 0.004 & 1.705 & $1.087-2.674$ & 0.02 \\
pStage & $320 / 92$ & $(1,2 / 3)$ & 3.647 & $2.415-5.509$ & $<0.001$ & 2.16 & $1.218-3.758$ & 0.008 \\
CEA & $318 / 94$ & $(<5.0 / \geq 5.0)$ & 2.245 & $1.467-3.436$ & $<0.001$ & 1.964 & $1.251-3.083$ & 0.003 \\
CRP & $350 / 62$ & $(<0.5 />0.5)$ & 2.452 & $1.555-3.865$ & $<0.001$ & 1.284 & $0.750-2.199$ & 0.361 \\
SII & $307 / 105$ & $(<661.9 / \geq 661.9)$ & 2.669 & $1.755-4.060$ & $<0.001$ & 1.055 & $0.510-2.182$ & 0.885 \\
NLR & $268 / 144$ & $(<2.529 / \geq 2.529)$ & 2.465 & $1.630-3.728$ & $<0.001$ & 1.571 & $0.804-3.069$ & 0.187 \\
PLR & $349 / 63$ & $(<212.1 / \geq 212.1)$ & 2.671 & $1.680-4.244$ & $<0.001$ & 1.417 & $0.780-2.573$ & 0.252 \\
Postoperative & $290 / 122$ & $($ absent/present) & 1.982 & $1.300-3.020$ & 0.002 & 1.474 & $0.934-2.325$ & 0.095 \\
complications & & & & & & & & \\
Adjuvant & $298 / 114$ & $($ No/Yes $)$ & 1.814 & $1.193-2.757$ & 0.005 & 0.979 & $0.587-1.634$ & 0.936 \\
\hline
\end{tabular}

Table 3: Relationships between SII and clinicopathological features in inflammation-stratified gastric cancer patients

\begin{tabular}{|c|c|c|c|c|c|c|c|c|}
\hline \multirow{4}{*}{ Characteristics } & \multicolumn{5}{|c|}{ Without inflammation } & \multirow{2}{*}{\multicolumn{3}{|c|}{$\begin{array}{c}\text { With inflammation } \\
\text { SII }\end{array}$}} \\
\hline & \multirow{3}{*}{$\begin{array}{l}\text { Total } \\
\text { patients }\end{array}$} & \multicolumn{2}{|c|}{ SII } & \multirow[b]{3}{*}{$p$ value } & \multirow{3}{*}{$\begin{array}{c}\text { Total } \\
\text { patients }\end{array}$} & & & \\
\hline & & $<661.9$ & $\geq 661.9$ & & & $<661.9$ & $\geq 661.9$ & \multirow{2}{*}{$p$ value } \\
\hline & & $(n=274)$ & $(n=76)$ & & & $(n=33)$ & $(n=29)$ & \\
\hline Age (years) & & $69.5(36-91)$ & $73.5(43-89)$ & 0.081 & & $74(56-82)$ & $74(61-90)$ & 0.374 \\
\hline Gender & & & & 0.975 & & & & 0.546 \\
\hline Male & 240 & 188 & 52 & & 47 & 24 & 23 & \\
\hline Female & 110 & 86 & 24 & & 15 & 9 & 6 & \\
\hline ASA & & & & 0.044 & & & & 0.018 \\
\hline 1 & 24 & 20 & 4 & & 0 & 0 & 0 & \\
\hline 2 & 302 & 240 & 62 & & 47 & 29 & 18 & \\
\hline 3 & 24 & 14 & 10 & & 15 & 4 & 11 & \\
\hline BMI & & $22.5(14.7-40.4)$ & $21.9(15.4-32.5)$ & 0.067 & & $22.0(15.6-28.3)$ & $21.5(14.0-29.8)$ & 0.703 \\
\hline WBC & & $5495(1830-9280)$ & $6480(4040-10300)$ & $<0.001$ & & $6130(3180-8260)$ & $6730(3510-13700)$ & 0.066 \\
\hline Lymphocyte & & $1780(470-3780)$ & $1220(230-2270)$ & $<0.001$ & & $1660(800-3180)$ & $1220(450-2500)$ & 0.002 \\
\hline Neutroohil & & $3125(1100-6190)$ & $4530(2710-7537)$ & $<0.001$ & & $3492(1710-5770)$ & $4440(2650-8494)$ & $<0.001$ \\
\hline Platelet & & $207(58-460)$ & $248(123-665)$ & $<0.001$ & & $199(94-343)$ & $295(119-726)$ & $<0.001$ \\
\hline Tumor size (mm) & & $38(4-180)$ & $50(5-150)$ & 0.015 & & $50(3-126)$ & $65(12-170)$ & 0.244 \\
\hline Differentiation & & & & 0.501 & & & & 0.298 \\
\hline Well & 73 & 59 & 14 & & 8 & 6 & 2 & \\
\hline Moderate & 123 & 92 & 31 & & 29 & 16 & 13 & \\
\hline Poor & 154 & 123 & 31 & & 25 & 11 & 14 & \\
\hline Depth of tumor & & & & 0.088 & & & & 0.002 \\
\hline T1a-1b & 200 & 166 & 34 & & 18 & 14 & 4 & \\
\hline 2 & 48 & 36 & 12 & & 6 & 6 & 0 & \\
\hline 3 & 46 & 33 & 13 & & 13 & 4 & 9 & \\
\hline $4 a-4 b$ & 56 & 39 & 17 & & 25 & 9 & 16 & \\
\hline Lymph node metastasis & & & & 0.662 & & & & 0.047 \\
\hline
\end{tabular}




\begin{tabular}{|c|c|c|c|c|c|c|c|c|}
\hline No & 245 & 196 & 49 & & 29 & 19 & 10 & \\
\hline N1 & 49 & 29 & 11 & & 10 & 7 & 3 & \\
\hline N2 & 30 & 23 & 7 & & 15 & 4 & 11 & \\
\hline N3 & 35 & 26 & 9 & & 8 & 3 & 5 & \\
\hline Pathological stage & & & & 0.088 & & & & 0.001 \\
\hline $1 a-1 b$ & 226 & 185 & 41 & & 21 & 18 & 3 & \\
\hline $2 a-2 b$ & 59 & 43 & 16 & & 14 & 6 & 8 & \\
\hline $3 a-3 c$ & 65 & 46 & 19 & & 27 & 9 & 18 & \\
\hline CEA antigen (ng/ml) & & $3.1(0.7-106)$ & $3.6(0.8-163.3)$ & 0.041 & & $3.7(1.0-76.3)$ & $2.8(1.3-29.5)$ & 0.323 \\
\hline NLR & & $1.784(0.648-6.894)$ & $3.71(1.968-16.043)$ & $<0.001$ & & $\begin{array}{c}2.196 \\
(0.881-3.667)\end{array}$ & $\begin{array}{c}3.656 \\
(1.880-6.909)\end{array}$ & $<0.001$ \\
\hline PLR & & $117.0(46.7-276.9)$ & $202.1(99.4-708.7)$ & $<0.001$ & & $\begin{array}{c}115.2 \\
(43.8-221.3)\end{array}$ & $234.0(119.6-992.7)$ & $<0.001$ \\
\hline $\begin{array}{l}\text { Postoperative } \\
\text { complications }\end{array}$ & & & & 0.955 & & & & 0.024 \\
\hline absent & 257 & 201 & 56 & & 33 & 22 & 11 & \\
\hline present & 93 & 73 & 20 & & 29 & 11 & 18 & \\
\hline Adjuvant chemotherapy & & & & 0.338 & & & & 0.513 \\
\hline Yes & 91 & 68 & 23 & & 23 & 11 & 12 & \\
\hline No & 259 & 206 & 53 & & 39 & 22 & 17 & \\
\hline
\end{tabular}

Table 4: Univariate and multivariate analyses for OS in inflammation-stratified gastric cancer patients

\begin{tabular}{|c|c|c|c|c|c|c|c|c|c|c|c|c|c|c|c|}
\hline \multirow[b]{3}{*}{ Variables } & \multicolumn{8}{|c|}{ Without inflammation } & \multicolumn{7}{|c|}{ With inflammation } \\
\hline & \multirow[b]{2}{*}{$\begin{array}{l}\text { Patients } \\
(n=350)\end{array}$} & \multirow[b]{2}{*}{$\begin{array}{c}\text { Category or } \\
\text { characteristics }\end{array}$} & \multicolumn{3}{|c|}{ Univariate } & \multicolumn{3}{|c|}{ Multivariate } & \multirow[b]{2}{*}{$\begin{array}{c}\text { Patients } \\
(n=62)\end{array}$} & \multicolumn{3}{|c|}{ Univariate analysis } & \multicolumn{3}{|c|}{ Multivariate analysis } \\
\hline & & & HR & $95 \% \mathrm{CI}$ & $p$ value & HR & $95 \% \mathrm{CI}$ & $\begin{array}{c}p \\
\text { value }\end{array}$ & & HR & $95 \% \mathrm{CI}$ & $\begin{array}{c}p \\
\text { value }\end{array}$ & HR & $95 \% \mathrm{CI}$ & $\begin{array}{c}p \\
\text { value }\end{array}$ \\
\hline Age & $100 / 250$ & $(<65 / \geq 65)$ & 2.395 & $1.221-4.700$ & 0.011 & 2.088 & $1.033-4.221$ & 0.04 & $12 / 50$ & 1.511 & $0.519-4.396$ & 0.449 & & & \\
\hline Gender & $(110 / 240)$ & (female/male) & 1.266 & $0.735-2.181$ & 0.395 & & & & $15 / 47$ & 1.487 & $0.559-3.955$ & 0.427 & & & \\
\hline BMI & $318 / 32$ & $(>18.5 /<18.5)$ & 1.63 & $0.830-3.201$ & 0.156 & & & & $54 / 8$ & 3.208 & $1.050-9.803$ & 0.041 & 5.055 & $1.442-17.726$ & 0.011 \\
\hline ASA & $326 / 24$ & $(<3 / \geq 3)$ & 3.3 & $1.554-7.010$ & 0.002 & 2.339 & $1.026-5.332$ & 0.043 & $47 / 15$ & 3.711 & $1.689-8.152$ & 0.001 & 3.403 & $1.388-8.342$ & 0.007 \\
\hline Tumor size & $219 / 131$ & $(<5 / \geq 5)$ & 2.515 & $1.533-4.126$ & $<0.001$ & 1.473 & $0.813-2.667$ & 0.201 & $25 / 37$ & 1.109 & $0.502-2.452$ & 0.798 & & & \\
\hline Differentiation & $195 / 55$ & $\begin{array}{c}\text { (well \& mod/ } \\
\text { poor) }\end{array}$ & 2.297 & $1.384-3.821$ & 0.001 & 1.748 & $1.014-3.013$ & 0.044 & $37 / 25$ & 1.317 & $0.602-2.880$ & 0.49 & & & \\
\hline pStage & $285 / 65$ & $(1,2 / 3)$ & 3.951 & $2.421-6.447$ & $<0.001$ & 2.114 & $1.103-4.050$ & 0.024 & $35 / 27$ & 1.989 & $0.898-4.273$ & 0.091 & & & \\
\hline CEA & $273 / 77$ & $(<5.0 / \geq 5.0)$ & 2.291 & $1.388-3.781$ & 0.001 & 1.703 & $0.994-2.917$ & 0.053 & $47 / 17$ & 2.12 & $0.932-4.822$ & 0.073 & & & \\
\hline SII & $274 / 76$ & $(<661.9 / \geq 661.9)$ & 2.229 & $1.330-3.735$ & 0.002 & 0.783 & $0.350-1.751$ & 0.551 & $33 / 29$ & 2.776 & $1.227-6.279$ & 0.014 & 4.208 & $1.189-14.887$ & 0.026 \\
\hline NLR & $241 / 109$ & $(<2.529 / \geq 2.529)$ & 2.172 & $1.327-3.557$ & 0.002 & 1.755 & $0.848-3.630$ & 0.13 & $27 / 35$ & 2.362 & $1.016-5.490$ & 0.046 & 0.602 & $0.159-2.274$ & 0.454 \\
\hline PLR & $306 / 44$ & $(<212.1 / \geq 212.1)$ & 2.921 & $1.655-5.157$ & $<0.001$ & 2.068 & $1.007-4.245$ & 0.058 & $43 / 19$ & 1.467 & $0.649-3.316$ & 0.358 & & & \\
\hline $\begin{array}{l}\text { Postoperative } \\
\text { complications }\end{array}$ & $257 / 93$ & (absent/present) & 1.645 & $0.977-2.771$ & 0.061 & & & & $33 / 29$ & 2.126 & $0.970-4.661$ & 0.060 & & & \\
\hline Adjuvant & $259 / 91$ & (No/Yes) & 2.794 & $1.714-4.554$ & $<0.001$ & 1.355 & $0.749-2.451$ & 0.315 & $39 / 23$ & 0.411 & $0.165-1.025$ & 0.057 & & & \\
\hline
\end{tabular}

On univariate analyses for patients with inflammation, OS was found to be significantly associated with high BMI $(p=0.041)$, poor ASAPS $(p=0.001)$, high SII $(p=0.014)$, and high NLR $(p=0.046)$. Multivariate analysis revealed that BMI (HR: 5.055, 95.0\% CI: $1.442-17.726 ; p=0.011)$, ASA-PS (HR: $3.403,95.0 \%$ CI: $1.388-8.342 ; p=0.007$ ), and SII (HR: 4.208, 95.0\% CI: $1.189-14.887 ; p=0.026$ ) were the independent prognostic factors of OS.

\section{SII and OS across all patients}

Patients with a low SII had a 3-year OS rate of $86.5 \%$ and a 5 -year OS rate of $78.9 \%$. In patients with a high SII, the 3-and 5-year OS rates were $74.1 \%$ and $54.7 \%$, respectively. The log-rank test demonstrated that patients with a high SII had significantly worse OS than those with a low SII $(p<0.001)$ (Figure 1).

\section{SII and OS in inflammation-stratified groups}

In patients without inflammation, the Kaplan-Meier analysis revealed that the 3- and 5-year OS rates in patients with a low SII were $87.5 \%$ and $79.9 \%$, respectively, while for patients with a high SII the 3- and 5-year OS rates were $84.3 \%$ and $63.0 \%$, respectively. The log-rank test demonstrated that patients with a high SII had significantly worse OS than those with a low SII $(p=0.002)$ (Figure 2A). 
In patients with inflammation, the KaplanMeier analysis revealed that the 3- and 5-year OS rates in patients with a low SII were $79.0 \%$ and $70.7 \%$, respectively, and those in patients with a high SII the 3and 5-year OS rates were $50.0 \%$ and $35.8 \%$, respectively. The log-rank test demonstrated that patients with a high SII had significantly worse OS than those with a low SII $(p=0.011)$ (Figure 2B).

\section{Predictive ability of SII, NLR, and PLR for OS in inflammation-stratified patients}

In patients without inflammation, AUCs for SII, NLR, and PLR were $0.565,0.584$ and 0.577 , respectively (Figure 3A). Additionally, AUCs for SII, NLR and PLR in patients with inflammation were $0.614,0.603$ and 0.565 , respectively. SII consistently had a higher AUC value compare to NLR and PLR in gastric cancer patients with inflammation (Figure 3B).

\section{DISCUSSION}

Previous studies have compared the prognostic impact of inflammation-based parameters, including SII and NLR, in lung, pancreas, ovary, or colon cancer [10-13]. To our knowledge, however, there are no studies on the most suitable parameter for predicting long-term outcomes in gastric cancer. Thus, in this study, we aimed to clarify the clinical and prognostic values of preoperative systemic inflammatory indicators, including SII, NLR, and PLR, in gastric cancer patients who underwent curative laparoscopic gastrectomy.

Neutrophils play crucial roles in the pathogenesis of cancer by enhancing the proliferation, invasion, and metastasis of cancer cells via the release of cytokines and chemokines such as interleukin (IL)-6 and tumor necrosis factor- $\alpha$ (TNF- $\alpha)$. Furthermore, these inflammatory cytokines/chemokines promote angiogenesis and cellular DNA damage, inhibit apoptosis, and protect cancer cells

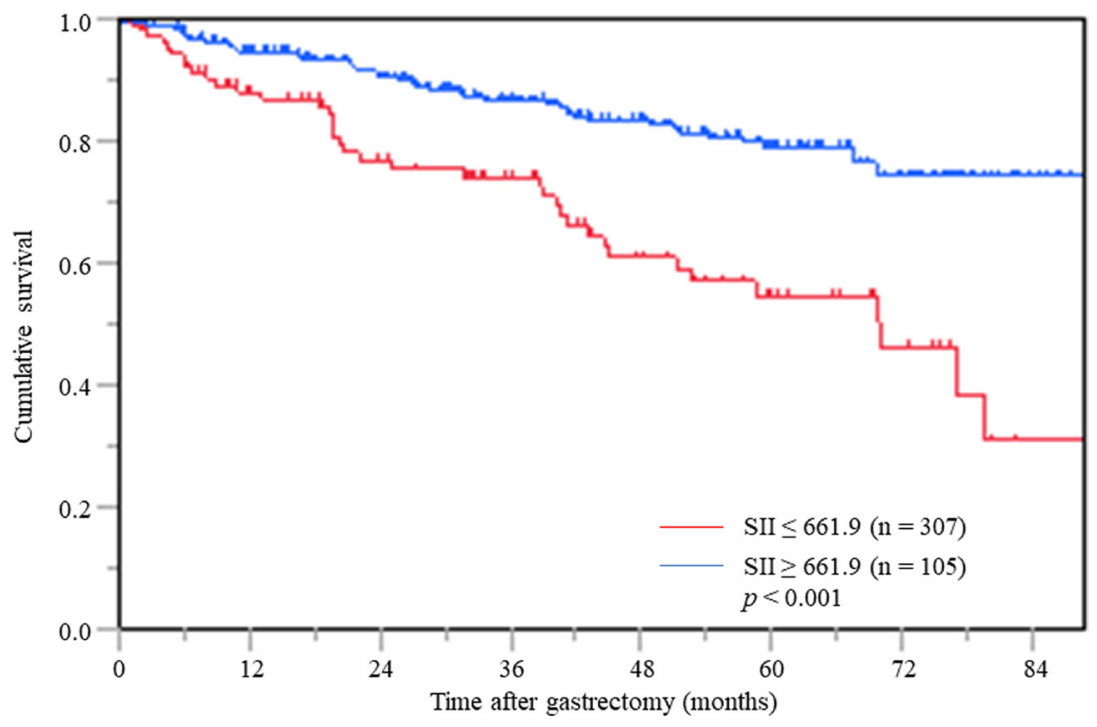

Figure 1: Postoperative OS based on SII in overall gastric cancer patients.
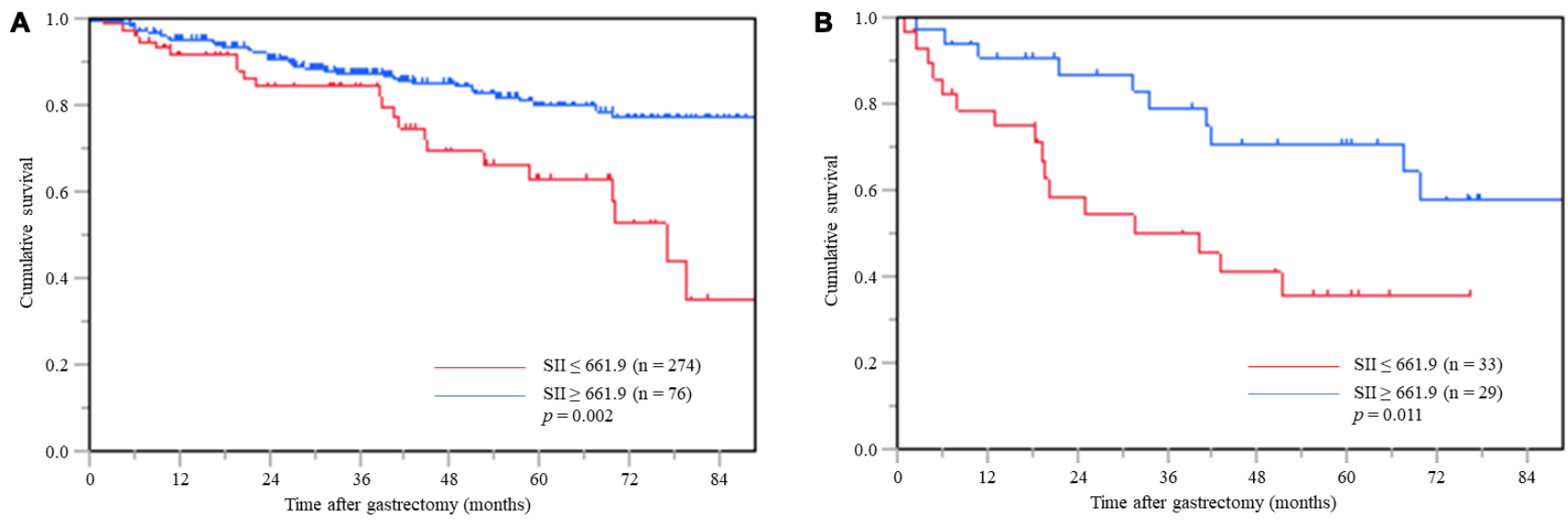

Figure 2: Postoperative OS based on SII in gastric cancer patients without inflammation (A) and with inflammation (B). 
from immune surveillance. As a result, an increasing number of neutrophils can establish a favorable tumor microenvironment and then promote tumor progression $[14,15]$.

Lymphocytes have an important role in tumor immune surveillance and defense against cancer by inducing cytotoxic cell death and inhibiting tumor cell proliferation and migration. Lymphocytes also block the proliferation and migration of cancer cells by secreting cytokines, such as interferon- $\gamma$ and TNF- $\alpha$. Thus, lymphocytes can eliminate cancer cells through cellular and humoral immune mechanisms $[16,17]$.

Platelets have been proven to induce epithelialmesenchymal transition via platelet-derived transforming growth factor- $\beta$ and direct platelet-tumor cell contact. The complex interaction between tumor cells and platelets favors distant metastasis of tumor cells and allows circulating tumor cells to escape from the host's immune surveillance [18, 19].

Based on the facts mentioned above, previous validations had warranted NLR and PLR, which consist of two types of inflammatory cells, to be well associated with cancer cell behavior and prognosis [10-13]. SII, which comprises 3 peripheral blood parameters, has recently been shown to be a more objective and reliable biomarker for predicting prognosis of cancer patients because it comprehensively reflects both the balance between host inflammatory and immune responses $[5,10]$.

In our study, a high SII was significantly associated with a larger tumor size, deeper invasion, increased lymph node metastasis, and advanced TNM stage in gastric cancer, indicating a more aggressive tumor phenotype. Therefore,

\section{A}

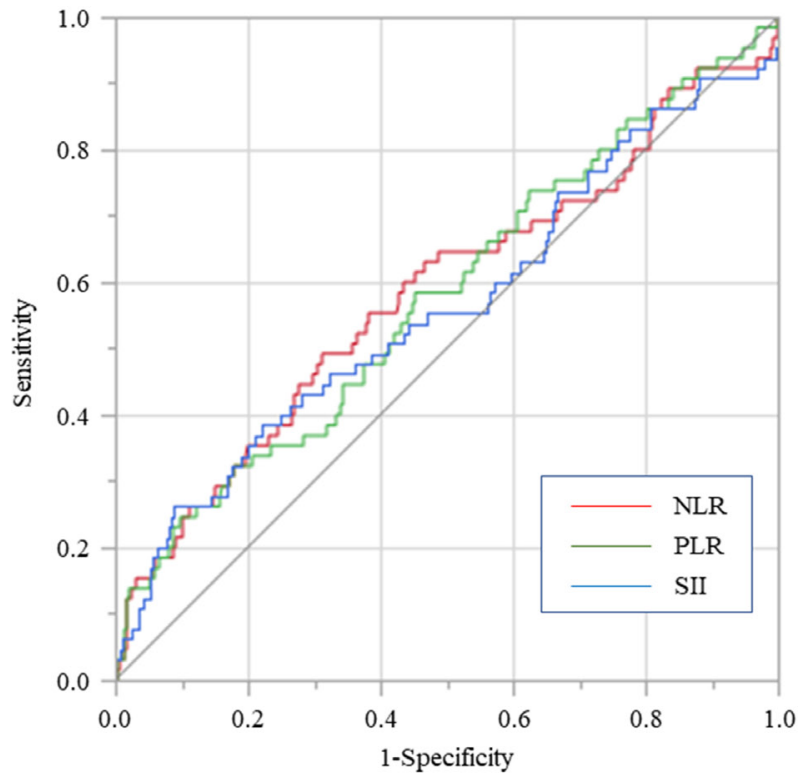

SII could be a beneficial complement to TNM stage in the preoperative prediction of survival in cancer patients.

Virchow has reported that inflammatory reactions and cancer, several studies have found that CRP level is a prognostic factor in a variety of cancer [20]. Systemic inflammatory proteins represented by CRP are synthesized by hepatocytes and induced by proinflammatory cytokines, particularly IL-1, IL-6 and TNF- $\alpha[21,22]$. CRP is directly associated with acceleration of angiogenesis, which enhances the progression and metastasis of malignant tumors and contributes to the progression of cancer. CRP is one of the most frequently used serum markers to evaluate prognosis of cancer, but it lacks specificity and could be elevated in a number of systemic influences such as infections, surgery, and connective tissue disease [23-26]. CRP is one of the most frequently used serum markers to assess cancer prognosis, but due to lack of specificity, several studies have been reported, including Glasgow Prognostic Score (Glasgow Prognostic Score (GPS) and CRP/albumin ratio (CAR), which combine CRP and albumin [27-30]. Although the majority of gastric cancer patients have normal CRP, the prognosis prediction by combination of CRP and SII, which comprehensively reflect the balance of host immune and inflammatory status, is important in determining the individualized surveillance and optimized therapeutic strategy to improve prognosis. Inflammatory stratification analysis was performed based on a reference $0.5 \mathrm{mg} / \mathrm{dl}$ for CRP, a component of modified GPS reported by Miki et. al [31, 32]. In our inflammationbased cohort, NLR, PLR, and SII were associated with OS in univariate Cox analyses, but they inversely failed

\section{B}

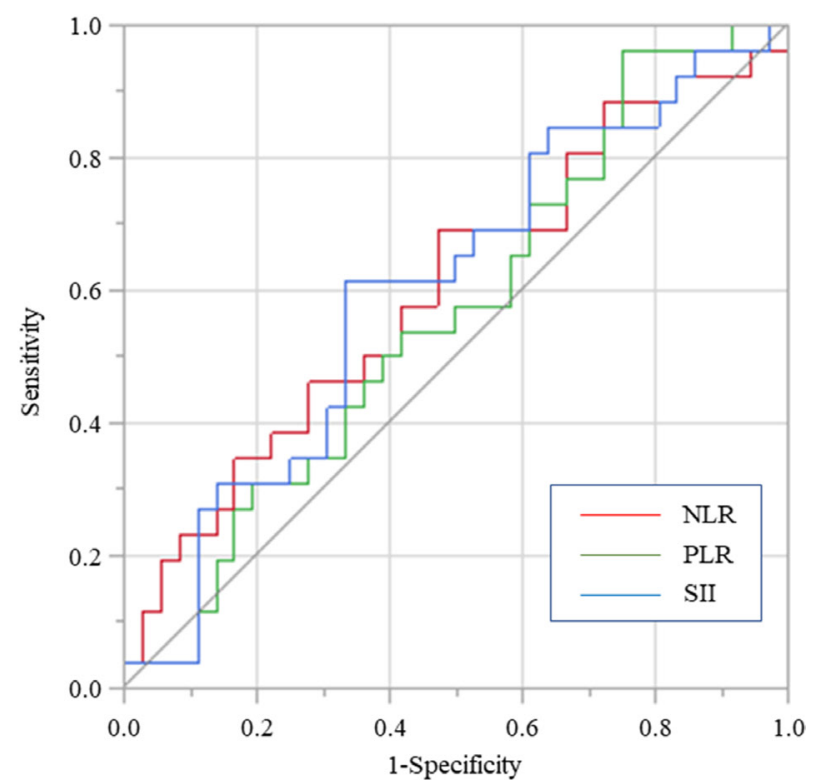

Figure 3: Predictive abilities of SII, NLR, and PLR for OS examined using ROC curve analysis in gastric cancer patients without inflammation (A) and with inflammation (B). 
to achieve statistical significance in patient without inflammation on multivariate analysis and TNM staging remained independent factor at multivariate. On the other hand, only SII remained as an independent factor on multivariate analysis in patients with inflammation, inversely TNM staging was not associated with prognosis in patients with inflammation. CRP alone is unlikely to be a cancer-specific prognostic predictor, but in patients with inflammation, it was possible to predict cancer-specific prognosis when evaluated in combination with SII. In addition, the comparison of AUC using ROC analysis demonstrated that SII was superior to NLR and PLR for predicting OS after surgery in gastric cancer patients with inflammation. In terms of results, the stratification of prognostic prediction based on SII value is rational especially in patients with non-specific inflammation represented by CRP.

Several limitations of this study should be acknowledged. First, this study was conducted with a small sample size in a single institution, which accounts for the lack of statistical power. Second, there are no universal standard cutoff values for inflammation indices. Most studies determined individual cutoff values by their relevance and significance. As a result, there is a wide range of cutoff values for these indices. Significant cutoff values for inflammatory indices should be verified in properly designed multicentric, independent cohort patients before adopting SII as a predictive biomarker in clinical practice. Third, monitoring of SII during perioperative therapy may provide more important information about the status of systemic inflammatory and immune response as well as therapeutic benefit. In this study, we failed to evaluate postoperative dynamic changes in the SII values. Finally, although SII is a useful and readily available routine blood data, the biological and molecular mechanisms that explain the prognostic predictive nature of SII have not been examined. Furthermore, in overall patients, multivariate analysis showed that SII was an independent prognostic factor for cancer-specific survival, but inflammation stratified analysis did not confirm that SII was an independent prognostic factor for cancer-specific survival (CSS), as only patients without inflammation were significantly associated with CSS (data unshown).

In summary, this study showed that preoperative SII is the most significant prognostic biomarker for OS, especially in patients with gastric cancer with inflammation, when compared to two-factor markers such as NLR and PLR. In the future, properly designed prospective studies should confirm the more significant prognostic value of SII in gastric cancer.

\section{MATERIALS AND METHODS}

\section{Patients}

This retrospective study evaluated 412 consecutive patients who underwent curative laparoscopic gastrectomy for histologically verified gastric adenocarcinoma at our institution between January 2010 and December 2017. Exclusion criteria were as follows: neoadjuvant chemotherapy; active infection within 1 month before

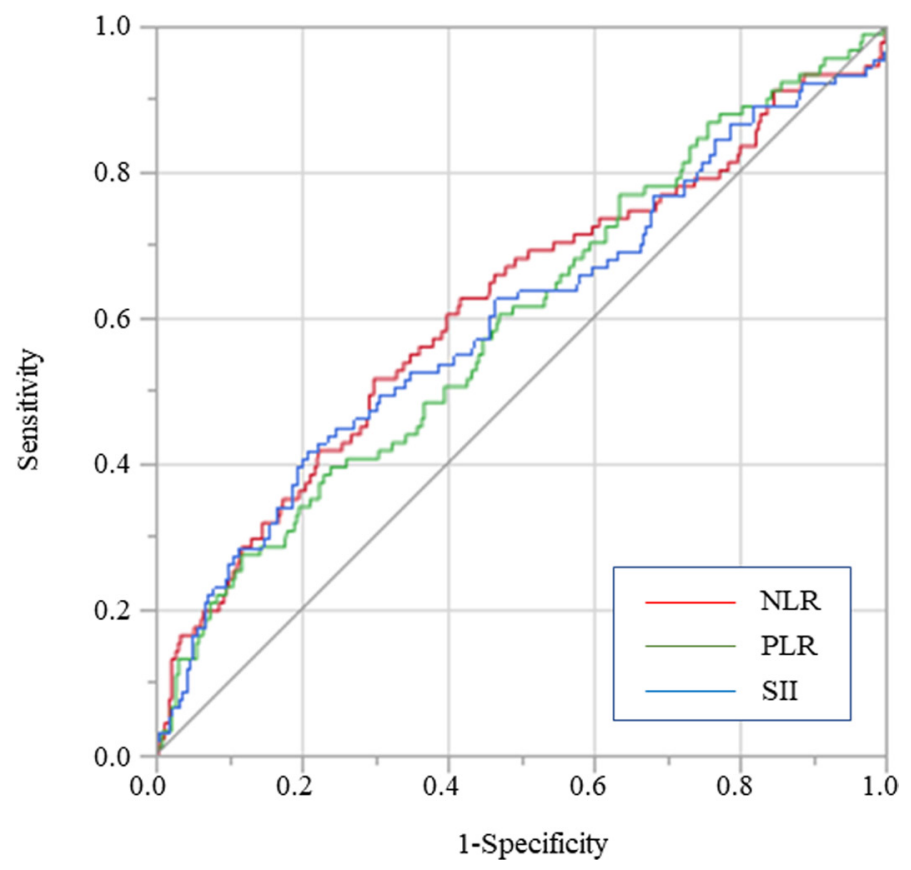

Figure 4: Predictive abilities of SII, NLR, and PLR for OS examined using ROC curve analysis in overall gastric cancer patients. 
surgery; inflammatory, bone marrow, hematological, or autoimmune disease; and a history of other malignancies within the preceding 5 years.

The extent of the gastrectomy and lymph node dissection were determined in accordance with the Japanese Gastric Cancer Treatment Guidelines (version 4) [33]. Similarly, postoperative adjuvant and post recurrence chemotherapy were administered according to the guidelines [33]. Postoperative complications were evaluated according to the Clavien-Dindo (CD) classification and serious complications were defined as grade II or higher [34]. Clinicopathological classification was assessed according to the International Union Against Cancer Tumor, Node, Metastasis (TNM) classification (seventh edition) [9].

The retrospective protocol of this study was approved by the Ethical Review Board of Shimane University, Faculty of Medicine (Shimane, Japan), and the study is registered with the University Hospital Medical Information Network Clinical Trials Registry (UMIN000030472). The requirement for written informed consent was waived because of the retrospective nature of the study.

\section{Hematological parameter calculation}

Preoperative complete blood count (CBC) and blood differential count were derived within 7 days prior to surgery from each patient. The SII was defined as follows: SII $=$ platelet count $\times$ neutrophil/lymphocyte count. The NLR and PLR were defined as follows: NLR = neutrophil/ lymphocyte count and PLR = platelet/lymphocyte count.

The receiver operating characteristic (ROC) curve was employed in determining the optimal cutoff value for each inflammation-based index for predicting overall survival (OS) after surgery. The optimal cutoff values were 661.9 for SII, 2.529 for NLR, and 212.1 for PLR, respectively. Consequently, patients were divided into a low or high group according to the individual optimal cutoff values for SII, NLR, and PLR. The area under the curves (AUC) for SII, NLR and PLR were 0.599, 0.613 and 0.593 , respectively (Figure 4 ).

\section{Follow-up after surgery}

Patients were carefully followed up after surgery every 3 months for 2 years, and then every 6 months from years 3 to 5 . The routine assessment for recurrence included blood examination, abdominal ultrasonography, chest X-ray imaging, and/or computed tomography. The OS was calculated from the date of surgical resection to the date of death from any cause or the date of last followup. The median follow-up duration was 35.9 months (range: 2.7-96.6 months).

\section{Statistical analysis}

The differences between the categorical variables were evaluated by using the Chi-squared test or Fisher's exact test. The Kaplan-Meier method was used to plot OS after surgery. The differences between survival curves were evaluated via the log-rank test. Cox proportional hazards regression models and Hazard ratios were calculated to test differences between groups. Variables with a $p$-value $<0.05$ following univariate analysis were subsequently subject to multivariate logistic regression analysis. All statistical analyses were performed using JMP software (version 15 for Windows; SAS Institute) and a $p$-value $<0.05$ was considered as statistically significant.

\section{Abbreviations}

ASA-PS: American Society of Anesthesiologists physical status; AUC: area under the curve; BMI: body mass index; CAR: CRP/albumin ratio (CAR), CD: Clavien-Dindo; CEA: carcinoembryonic antigen; CRP: C-reactive protein; CSS: cancer-specific survival; GPS: Glasgow Prognostic Score; HR: hazard ratio; IL: interleukin; NLR: neutrophil/lymphocyte ratio; OS: overall survival; PLR: platelet/lymphocyte ratio; RBC: red blood cell; SII: systemic immune-inflammation index; ROC: receiver operating characteristic; TNF- $\alpha$ : tumor necrosis factor-a; TNM: tumor-node-metastasis; WBC: white blood cell count.

\section{Author contributions}

NH was the lead author and conceived this study. TM, YF, SK, and RH conducted this research. TY, YK, and TT collected the data, performed the analyses, and drafted the manuscript. YT reviewed the paper and the surgery technique. All authors read and approved the final manuscript.

\section{ACKNOWLEDGMENTS}

None.

\section{CONFLICTS OF INTEREST}

None of the authors have any conflicts of interest.

\section{FUNDING}

This study received no external sources of funding.

\section{REFERENCES}

1. Wang F, Arun P, Friedman J, Chen Z, Van Waes C. Current and potential inflammation targeted therapies in head and neck cancer. Curr Opin Pharmacol. 2009; 9:389-95. https:// doi.org/10.1016/j.coph.2009.06.005. [PubMed]

2. Zhang J, Wang P, Ouyang H, Yin J, Liu A, Ma C, Liu L. Targeting cancer-related inflammation: chinese herbal 
medicine inhibits epithelial-to-mesenchymal transition in pancreatic cancer. PLoS One. 2013; 8:e70334. https://doi. org/10.1371/journal.pone.0070334. [PubMed]

3. Candido J, Hagemann T. Cancer-related inflammation. J Clin Immunol. 2013; 33:S79-84. https://doi.org/10.1007/ s10875-012-9847-0. [PubMed]

4. Azouz A, Razzaque MS, El-Hallak M, Taguchi T. Immunoinflammatory responses and fibrogenesis. Med Electron Microsc. 2004; 37:141-48. https://doi.org/10.1007/ s00795-004-0255-2. [PubMed]

5. Hu B, Yang XR, Xu Y, Sun YF, Sun C, Guo W, Zhang X, Wang WM, Qiu SJ, Zhou J, Fan J. Systemic immuneinflammation index predicts prognosis of patients after curative resection for hepatocellular carcinoma. Clin Cancer Res. 2014; 20:6212-22. https://doi.org/10.1158/1078-0432. CCR-14-0442. [PubMed]

6. Hirahara N, Matsubara T, Kawahara D, Nakada S, Ishibashi S, Tajima Y. Prognostic significance of preoperative inflammatory response biomarkers in patients undergoing curative thoracoscopic esophagectomy for esophageal squamous cell carcinoma. Eur J Surg Oncol. 2017; 43:493-501. https://doi.org/10.1016/j. ejso.2016.11.018. [PubMed]

7. Dutta S, Crumley AB, Fullarton GM, Horgan PG, McMillan DC. Comparison of the prognostic value of tumour- and patient-related factors in patients undergoing potentially curative resection of oesophageal cancer. World J Surg. 2011; 35:1861-66. https://doi.org/10.1007/s00268-0111130-7. [PubMed]

8. Feng JF, Huang Y, Chen QX. Preoperative platelet lymphocyte ratio (PLR) is superior to neutrophil lymphocyte ratio (NLR) as a predictive factor in patients with esophageal squamous cell carcinoma. World J Surg Oncol. 2014; 12:58. https://doi.org/10.1186/1477-7819-12-58. [PubMed]

9. Sobin L, Gospodarowicz M, Wittekind C, eds. International Union against Cancer (UICC). TNM classification of malignant tumors, 7th ed. New York: Wiley-Blackwell; 2010.

10. Wang Y, Li Y, Chen P, Xu W, Wu Y, Che G. Prognostic value of the pretreatment systemic immune-inflammation index (SII) in patients with non-small cell lung cancer: a meta-analysis. Ann Transl Med. 2019; 7:433. https://doi. org/10.21037/atm.2019.08.116. [PubMed]

11. Kubo H, Murakami T, Matsuyama R, Yabushita Y, Tsuchiya N, Sawada Y, Homma Y, Kumamoto T, Endo I. Prognostic Impact of the Neutrophil-toLymphocyte Ratio in Borderline Resectable Pancreatic Ductal Adenocarcinoma Treated with Neoadjuvant Chemoradiotherapy Followed by Surgical Resection. World J Surg. 2019; 43:3153-60. https://doi.org/10.1007/ s00268-019-05159-9. [PubMed]

12. Lu C, Zhou L, Ouyang J, Yang H. Prognostic value of lymphocyte-to-monocyte ratio in ovarian cancer: a metaanalysis. Medicine (Baltimore). 2019; 98:e15876. https:// doi.org/10.1097/MD.0000000000015876. [PubMed]
13. Silva TH, Schilithz AO, Peres WA, Murad LB. Neutrophillymphocyte ratio and nutritional status are clinically useful in predicting prognosis in colorectal cancer patients. Nutr Cancer. 2019; 1-10. https://doi.org/10.1080/01635581.201 9.1679198. [PubMed]

14. Oka Y, Murata A, Nishijima J, Ogawa M, Mori T. The mechanism of hepatic cellular injury in sepsis: an in vitro study of the implications of cytokines and neutrophils in its pathogenesis. J Surg Res. 1993; 55:1-8. https://doi. org/10.1006/jsre.1993.1100. [PubMed]

15. Jablonska E. Soluble IL-6 and TNF receptors release by polymorphonuclear and mononuclear cells in cancer patients. Neoplasma. 1999; 46:35-39. [PubMed]

16. Manna SK, Mukhopadhyay A, Aggarwal BB. IFN-alpha suppresses activation of nuclear transcription factors NF-kappa B and activator protein 1 and potentiates TNFinduced apoptosis. J Immunol. 2000; 165:4927-34. https:// doi.org/10.4049/jimmunol.165.9.4927. [PubMed]

17. Currier NL, Miller SC. TNF-alpha further augments natural killer cells when co-administered with an interferon inducer to irradiated, leukemic, bone-marrow-transplanted mice. Cancer Chemother Pharmacol. 2001; 47:185-86. https:// doi.org/10.1007/s002800000222. [PubMed]

18. Weima SM, van Rooijen MA, Mummery CL, Feijen A, Kruijer W, de Laat SW, van Zoelen EJ. Differentially regulated production of platelet-derived growth factor and of transforming growth factor beta by a human teratocarcinoma cell line. Differentiation. 1988; 38:20310. https://doi.org/10.1111/j.1432-0436.1988.tb00214.x. [PubMed]

19. Oku K, Tanaka A, Yamanishi H, Nishizawa Y, Matsumoto $\mathrm{K}$, Shiozaki H, Mori T. Effects of various growth factors on growth of a cloned human esophageal squamous cancer cell line in a protein-free medium. Anticancer Res. 1991; 11:1591-95. [PubMed]

20. Mantovani A, Romero P, Palucka AK, Marincola FM. Tumour immunity: effector response to tumour and role of the microenvironment. Lancet. 2008; 371:771-83. https:// doi.org/10.1016/S0140-6736(08)60241-X. [PubMed]

21. Okada Y, Takahashi A, Ohmiya H, Kumasaka N, Kamatani Y, Hosono N, Tsunoda T, Matsuda K, Tanaka T, Kubo M, Nakamura Y, Yamamoto K, Kamatani N. Genome-wide association study for c-reactive protein levels identified pleiotropic associations in the IL6 locus. Hum Mol Genet. 2011; 20:1224-31. https://doi.org/10.1093/hmg/ddq551. [PubMed]

22. Gelin J, Moldawer LL, Lönnroth C, Sherry B, Chizzonite $\mathrm{R}$, Lundholm K. Role of endogenous tumor necrosis factor alpha and interleukin 1 for experimental tumor growth and the development of cancer cachexia. Cancer Res. 1991; 51:415-21. [PubMed]

23. Helzlsouer KJ, Erlinger TP, Platz EA. C-reactive protein levels and subsequent cancer outcomes: results from a prospective cohort study. Eur J Cancer. 2006; 42:704-07. https://doi.org/10.1016/j.ejca.2006.01.008. [PubMed] 
24. Shimetani N, Shimetani K, Mori M. Clinical evaluation of the measurement of serum procalcitonin: comparative study of procalcitonin and serum amyloid a protein in patients with high and low concentrations of serum c-reactive protein. Scand J Clin Lab Invest. 2004; 64:469-74. https:// doi.org/10.1080/00365510410006658. [PubMed]

25. Nozoe T, Iguchi T, Adachi E, Matsukuma A, Ezaki T. Preoperative elevation of serum c-reactive protein as an independent prognostic indicator for gastric cancer. Surg Today. 2011; 41:510-13. https://doi.org/10.1007/s00595009-4297-x. [PubMed]

26. McMillan DC, Wotherspoon HA, Fearon KC, Sturgeon C, Cooke TG, McArdle CS. A prospective study of tumor recurrence and the acute-phase response after apparently curative colorectal cancer surgery. Am J Surg. 1995; 170:319-22. https://doi.org/10.1016/s0002-9610(99)802967. [ PubMed]

27. Hirahara N, Matsubara T, Hayashi H, Takai K, Fujii Y, Tajima Y. Impact of inflammation-based prognostic score on survival after curative thoracoscopic esophagectomy for esophageal cancer. Eur J Surg Oncol. 2015; 41:1308-15. https://doi.org/10.1016/j.ejso.2015.07.008. [PubMed]

28. Nakao Y, Yamashita YI, Arima K, Miyata T, Itoyama R, Yusa T, Umezaki N, Yamao T, Nakagawa S, Okabe H, Imai K, Chikamoto A, Baba H. Clinical usefulness of perioperative c-reactive protein/albumin ratio in patients with intrahepatic cholangiocarcinoma: a retrospective single institutional study. Anticancer Res. 2019; 39:2641-46. https://doi.org/10.21873/anticanres.13388. [PubMed]

29. Haruki K, Shiba H, Shirai Y, Horiuchi T, Iwase R, Fujiwara Y, Furukawa K, Misawa T, Yanaga K. The c-reactive protein to albumin ratio predicts long-term outcomes in patients with pancreatic cancer after pancreatic resection. World J Surg. 2016; 40:2254-60. https://doi.org/10.1007/s00268016-3491-4. [PubMed]

30. Inamoto T, Matsuyama H, Sakano S, Ibuki N, Takahara K, Komura K, Takai T, Tsujino T, Yoshikawa Y, Minami K, Nagao K, Inoue R, Azuma H. The systemic inflammationbased glasgow prognostic score as a powerful prognostic factor in patients with upper tract urothelial carcinoma. Oncotarget. 2017; 8:113248-57. https://doi.org/10.18632/ oncotarget.22641. [PubMed]

31. Toiyama Y, Miki C, Inoue Y, Tanaka K, Mohri Y, Kusunoki $\mathrm{M}$. Evaluation of an inflammation-based prognostic score for the identification of patients requiring postoperative adjuvant chemotherapy for stage II colorectal cancer. Exp Ther Med. 2011; 2:95-101. https://doi.org/10.3892/ etm.2010.175. [PubMed]

32. Inoue $Y$, Iwata $\mathrm{T}$, Okugawa $\mathrm{Y}$, Kawamoto A, Hiro J, Toiyama Y, Tanaka K, Uchida K, Mohri Y, Miki C, Kusunoki M. Prognostic significance of a systemic inflammatory response in patients undergoing multimodality therapy for advanced colorectal cancer. Oncology. 2013; 84:100-7. https://doi.org/10.1159/000343822. [PubMed]

33. Japanese Gastric Cancer Association. Japanese gastric cancer treatment guidelines 2014 (ver. 4). Gastric Cancer. 2017; 20:1-19. https://doi.org/10.1007/s10120-016-0622-4. [PubMed]

34. Clavien PA, Barkun J, de Oliveira ML, Vauthey JN, Dindo D, Schulick RD, de Santibañes E, Pekolj J, Slankamenac K, Bassi C, Graf R, Vonlanthen R, Padbury R, et al. The clavien-dindo classification of surgical complications: fiveyear experience. Ann Surg. 2009; 250:187-96. https://doi. org/10.1097/SLA.0b013e3181b13ca2. [PubMed] 